\title{
Spectral-Domain Optical Coherence Tomography Analysis in Syndromic and Nonsyndromic Forms of Retinitis Pigmentosa due to USH2A Genetic Variants
}

\author{
Leonardo Colombo ${ }^{a}$ Paolo Enrico Maltese ${ }^{b}$ Dario Romano ${ }^{a}$ Paolo Fogagnolo ${ }^{a}$ \\ Marco Castoric Giuseppe Marceddu ${ }^{d}$ Francesca Cristofolid $^{d}$ Marcella Percio $^{b}$

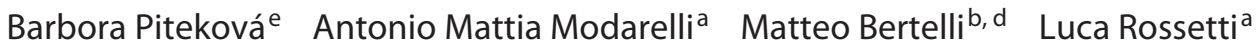 \\ aDepartment of Ophthalmology, ASST Santi Paolo e Carlo Hospital, University of Milan, Milan, Italy; ${ }^{b}$ MAGI'S Lab \\ s.r.l., Rovereto, Italy; 'Division of Medical Genetics, Fondazione IRCCS-Casa Sollievo della Sofferenza, San Giovanni

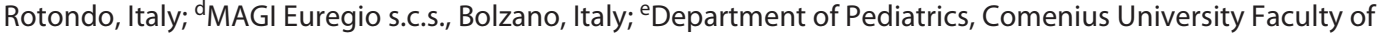 \\ Medicine, National Institute of Children's Diseases, Bratislava, Slovak Republic
}

\section{Keywords}

Spectral-domain optical coherence tomography .

Autosomal recessive retinitis pigmentosa - Usher syndrome . Usher syndrome type $2 \mathrm{~A}$

\begin{abstract}
Introduction: This study aimed to analyze macular structure by using spectral-domain optical coherence tomography (SD-OCT) in a cohort of patients affected by autosomal recessive retinitis pigmentosa and Usher syndrome, due to genetic variants in USH2A gene, and to correlate optical coherence tomography (OCT) parameters with functional and genetic data. Methods: The subjects of this study were 92 patients, 46 syndromic (Usher syndrome type lla [Ush2]) and 46 nonsyndromic (autosomal recessive RP [arRP]), with clinical and genetic diagnosis of USH2A-related retinal dystrophy, who underwent a complete ophthalmic examination and spectral-domain OCT analysis. The study focused on evaluating the differences between the 2 groups in the following parameters: best-corrected visual acuity (BCVA), ellipsoid zone (EZ) width, presence of epiretinal membrane (ERM), and cystic macular lesions (CMLs). Variants in USH2A
\end{abstract}

gene were divided into 3 categories, according to the expected impact (low/high) at protein level of the different variants on each allele. Results: BCVA and EZ width were significantly lower in Ush2 than in arRP patients $(p<0.0001$ and $p=0.001)$. ERM was detected in $34.8 \%(16 / 46)$ of arRP patients and in $65.2 \%(30 / 46)$ of Ush2 patients $(p=0.003)$. CML was detected in $17.4 \%(8 / 46)$ of arRP patients and $30.4 \%$ $(14 / 46)$ of Ush2 patients $(p=0.14)$. The allelic distribution was statistically different $(p=0.0003)$ by dividing the 2 diseases: for Ush2 patients it was $45.7 \%$ (high/high), 39.1\% (low/high) and 15.2\% (low/low); for arRP patients it was $8.7 \%$ (high/high), 56.5\% (low/high), and 34.8\% (low/low). The severity class of the variants significantly affected visual acuity and EZ width parameters ( $p=0.004$ and $p=0.002$, respectively). Conclusion: Retinal disease, as evaluated by means of SD-OCT, shows more advanced degeneration signs in the syndromic than the nonsyndromic form of retinal dystrophy related to USH2A gene. Variant types and allelic profiles are determining factors for the onset of syndromic features. However, since the 3 allelic profiles can be found in both Usher and RP patients, other factors must necessarily play a determining role.

(c) 2021 The Author(s)

Published by S. Karger AG, Basel karger@karger.com www.karger.com/ore

Karger $\stackrel{\text { ' }}{=}$

BOPEN ACCESS
(C) 2021 The Author(s)

Published by S. Karger AG, Basel

This is an Open Access article licensed under the Creative Commons Attribution-NonCommercial-4.0 International License (CC BY-NC) (http://www.karger.com/Services/OpenAccessLicense), applicable to the online version of the article only. Usage and distribution for commercial purposes requires written permission. 


\section{Introduction}

In the last decade, the advent of next-generation sequencing (NGS) technology allowed the identification of many disease-causing genes, thus increasing the understanding of genetic basis of retinitis pigmentosa (RP) $[1,2]$. To date, $>90$ genes are known in association with RP (https://sph.uth.edu/retnet/sum-dis.htm\#Agenes), and among these, the variants occurring in USH2A gene are the one most prevalently causing both autosomal recessive RP (arRP) or Usher syndrome type IIa (Ush2) [3-5].

The USH2A gene is located on chromosome 1q41abd and encodes for usherin, a basement membrane protein of many - but not all - tissues [6]. Two isoforms of this protein have been described, both expressed in the cells of photoreceptors and cochlear hairs. Isoform 1 (comprising 5,202 amino acids) is the longer one, translated by 72 exons, while the shorter isoform 2 (1,546 amino acids), is translated by the first 21 exons [7]. Usherin is a member of the USH2 complex involved in the maturation of the stereocilia of cochlear hair cells in the inner ear and in the long-term maintenance of photoreceptors in the retina [8], thus justifying its role in both arRP and Ush2. However, the relationship between the variants and the resulting phenotypes is still unclear [5].

New therapies - such as gene replacement or silencing, optogenetics, stem cells, retinal implants, and neuroprotective approaches - raised the need of increasingly accurate genotype-phenotype correlations and natural history studies [9-11]. Likewise, universally accepted guidelines are still required to objectively monitor the deterioration of retinal functions and structures, even in the short period.

Outer retinal evaluation by means of spectral-domain optical coherence tomography (SD-OCT) became part of clinical routine evaluation of RP patients. Ellipsoid zone (EZ) width is a well-accepted parameter to follow disease progression, also correlated with functional aspects like visual acuity (VA) and visual field (VF) [12-15]. The aim of our study was to provide new insights in the genotype-phenotype correlation of USH2A-related retinal dystrophies: for this purpose, we evaluated macular structure by means of SD-OCT in a large and well-defined cohort of patients affected by arRP and Ush2 due to USH2A genetic variants and we correlated the parameters with VA and the type of variants.

SD-OCT Analysis in USH2A-Associated

$\mathrm{RP}$ and Usher Syndrome

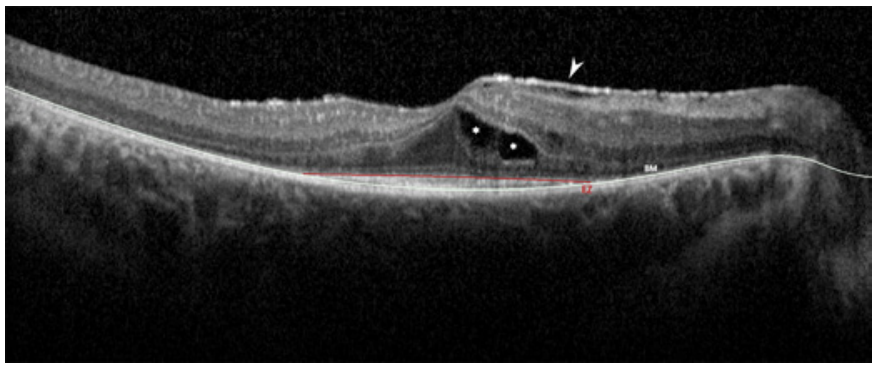

Fig. 1. SD-OCT scan of a patient affected by USH2A-related retinitis pigmentosa. The red line shows the EZ width; to measure it, the nasal and temporal boundaries were set where the EZ line meets Bruch's membrane line (as shown by the white line - BM). CML (asterisks) and ERM (arrow) were also evaluated. SD-OCT, spectral-domain optical coherence tomography; EZ, ellipsoid zone; ERM, epiretinal membrane; CML, cystic macular lesions; $\mathrm{VA}$, visual acuity.

\section{Materials and Methods}

\section{Study Population}

We recruited subjects with USH2A-related retinal dystrophies from outpatients of the Retinal Dystrophies department at the University Eye Clinic of ASST Santi Paolo e Carlo Hospital (Milan). RP diagnosis was based on the typical clinical signs (optic disc pallor, bone spicule pigmentation, and retinal vessel attenuation); the characteristic full-field electoretinographic patterns, which vary from reduced amplitudes of A and B waves in the early stages to nondetectable EGR in the advanced stages (as established by the International Society for Clinical Electrophysiology of Vision); VF constriction; and finally by the results of genetic analysis. We reviewed 101 electronic health records of patients with USH2A-related retinal dystrophies and we divided the sample into 2 groups: patients with sensorineural bilateral hearing loss were classified as Ush2, while the others were classified as nonsyndromic RP. The exclusion criteria were the lack of the psychophysical requirements to adequately understand the aim of the study, complete the required examinations, and provide the informed consent; and the presence of any other systemic or ocular conditions - unrelated with USH $2 A$ variants - that could worsen their vision abilities.

The patients meeting the criteria prospectively underwent the following evaluations: best-corrected visual acuity; slit-lamp examination of the anterior segment and indirect fundus biomicroscopy after pupil dilatation; and spectral-domain optical coherence tomography (OCT). Only good quality OCT scans (over $25 \mathrm{~dB}$ ) were used for the measurements: patients with cataract, other media opacities or any other condition (like nystagmus) affecting SDOCT scan quality were excluded.

The final cohort consisted of 46 patients with Ush2 and 46 with nonsyndromic RP. We included 3 pairs of siblings in the Usher group and 1 in the nonsyndromic group: for each pair, both siblings presented the same genotype and phenotype.

The study was compliant to the tenets of the Declaration of Helsinki and it was approved by the Ethics Committee of ASST Santi Paolo e Carlo Hospital. All participants were required to sign a written informed consent to take part to the study.

Ophthalmic Res 2022;65:180-195 
Table 1. Demographic characteristics of Usher syndrome and RP patients

\begin{tabular}{llllllll}
\hline Group (92 patients) & Sex & Age, years & Onset & VA & EZ & ERM, $n / N(\%)$ & CML, $n / N(\%)$ \\
\hline 46 RP & $24 \mathrm{M}$ & $50.33 \pm 12.83$ & $35.4 \pm 11.7$ & $0.653 \pm 0.281$ & $2,166.38 \pm 1,268.09$ & $9 / 24(37.50)$ & $5 / 24(20.83)$ \\
46 Usher lla & $22 \mathrm{~F}$ & $53.95 \pm 13.23$ & $34.9 \pm 15.2$ & $0.552 \pm 0.334$ & $1,754.32 \pm 1,554.55$ & $7 / 22(31.82)$ & $3 / 22(13.64)$ \\
& $18 \mathrm{M}$ & $41.78 \pm 13.96$ & $18.4 \pm 9.6$ & $0.431 \pm 0.287$ & $1,078.33 \pm 1,099.80$ & $14 / 18(77.78)$ & $5 / 18(27.78)$ \\
& $28 \mathrm{~F}$ & $45.67 \pm 13.68$ & $20.3 \pm 10.2$ & $0.535 \pm 0.310$ & $1,453.39 \pm 1,330.75$ & $16 / 28(57.14)$ & $9 / 28(32.14)$ \\
\hline
\end{tabular}

$R P$, retinitis pigmentosa; $V A$, visual acuity; EZ, ellipsoid zone; ERM, epiretinal membrane; CML, cystic macular lesion.

\section{Optical Coherence Tomography}

Structural retinal analysis was performed with SD-OCT and Heidelberg Eye Explorer (HEYEX) software (Heidelberg Engineering, Heidelberg, Germany). We analyzed single-line scans of $30^{\circ}$ crossing the fovea horizontally and centered using the automatic eye tracking system, obtained with Automatic Real-time Tracking (ART 100); a volume scan, performed using a $20^{\circ} \times 20^{\circ}$ scan field, with 49 B-scans (ART 12); and a radial layout, made of 6 scans of $20^{\circ}$ centered on the fovea (ART 12).

The external limiting membrane is the innermost of the 4 hyper-reflective bands on SD-OCT that can be identified in the human outer retina. The EZ is the second one, separated from the external limiting membrane by a hypo-reflective region called myoid zone. EZ was manually segmented for every single $30^{\circ}$ horizontal scan. Its nasal and temporal boundaries were defined to be the locations where this layer drops to the outer segment of photoreceptors and then its width was measured [12]. EZ width was manually measured by 2 experienced OCT-readers using the caliper of the HEYEX software; then the values obtained by the 2 physicians were averaged (shown in Fig. 1).

In addition, the presence/absence of cystic macular lesions (CMLs) and of the epiretinal membrane (ERM) was recorded analyzing volumetric scans. We defined CML as the presence of intraretinal hypo-reflective cystoid spaces in the central macula (1.5 $\mathrm{mm}$ diameter) that could be seen on at least 2 consecutive scans. CML was then divided - as proposed by [16] - into 3 categories: mild, moderate, and severe. ERM was defined as a hyper-reflective line above the internal limiting membrane and adherent to the inner retina, with or without distortion of the underlying retinal layers. OCT scans were obtained with dilated pupils (using 1\% tropicamide eye drops). Patients with poor OCT scan quality or prior vitreoretinal surgeries were excluded.

\section{Genetic Analysis}

Genetic evaluations were performed in MAGI's laboratories (MAGI'S Lab s.r.l.; Rovereto, and MAGI Euregio, Bolzano, Italy) starting from whole blood samples. The DNAs of the study subjects were extracted using a commercial kit (E.Z.N.A. Blood DNA kit Omega; Bio-Tek, Norcross, GA, USA) and used for subsequent analyses. All probands' samples were tested via NGS on an Illumina MiSeq personal sequencer (Illumina, San Diego, California) and a custom-designed multigene panel. Sequence variant calling and annotation were performed using an in-house bioinformatics pipeline, as described elsewhere [17, 18]. Sanger sequencing was used to validate NGS results and to perform segregation studies of selected variants in probands' relatives. Copy number variants were analyzed by multiplex ligation-dependent probe amplification (www.mrc-holland.com), using the Beckman Coulter CEQ 8000 sequencer (Beckmann Coulter, Milan, Italy) following the manufacturer instructions. The identified variants were searched in databases such as dbSNP (https://www.ncbi.nlm.nih.gov/snp/), ClinVar (https://www.ncbi.nlm.nih.gov/clinvar/), and Human Gene Mutation Database, professional version 2017.2 (https:// www.portal.biobase-international.com/hgmd/pro/). The pathogenicity of the variants was assessed following the American College of Medical Genetics and Genomics (ACMG) standard and guidelines [19] through the VarSome on-line software [20]. Genetic data were already available at the beginning of the present study and were retrospectively reviewed, to select only those patients who tested positive for USH2A gene from a larger sample of $\mathrm{RP}$ and Usher patients [3].

Variants in USH2A gene were divided into 3 groups based on the expected impact (low/high) at protein level of variants on each allele (maternal/paternal), according to the following classification: low/low, low/high, and high/high. Only missense variants were considered as having a low-impact. Nonsense, frameshift, in frame, splice site, and large deletions/insertions - such as copy number variants - were considered high impact variants.

\section{Statistical Analysis}

Analyses were performed on 1 eye per patient. Worst eye was defined as the one with lower VA or (in case of identical VA) larger EZ width. Data normality was tested according to KolmogorovSmirnov. Continuous variables were described as mean and standard deviations $( \pm)$, while categorical variables by using frequency. Covariance analysis was applied to the dataset by appointing age, sex, and allele status as covariates; in case of statistically significant results, the differences between groups were inspected using 2 -ways unpaired $t$ test (continuous data) or $\chi^{2}$ (categorical data). We also tested Pearson correlation between variables of interest. The analyses were performed using IBM SPSS (IBM Corp. Released 2017. IBM SPSS Statistics for Windows, Version 25.0.; IBM Corp., Armonk, NY, USA).

\section{Results}

We considered data from 92 patients affected by USH2A-related retinal dystrophy: 46 of them were classified as arRP and 46 as Ush2. In the arRP group, 24 sub- 


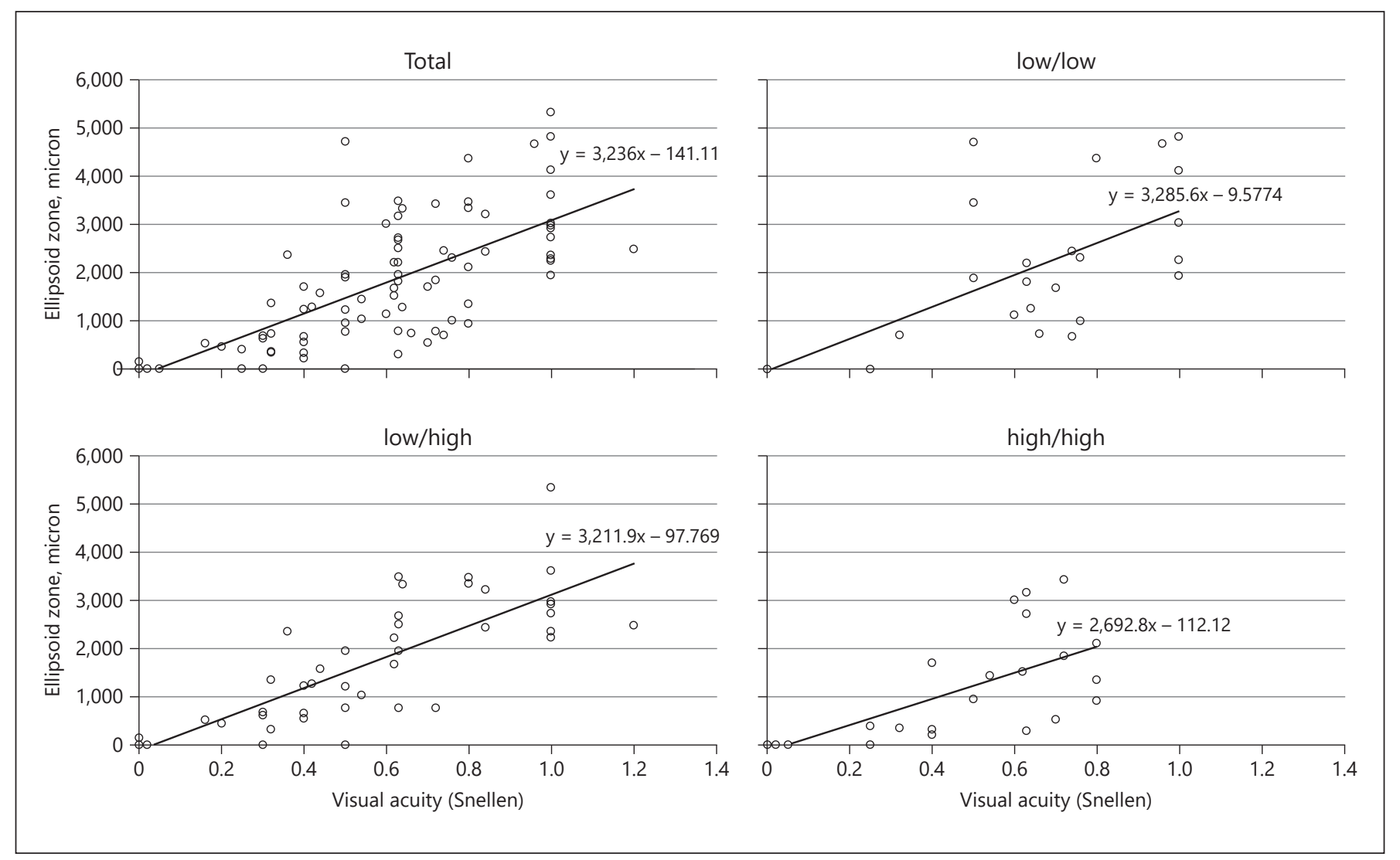

Fig. 2. Correlation between VA and EZ width in the whole cohort (total) or divided according to the genotype distribution by the expected variant impact (low/high) on each allele. VA, visual acuity; EZ, ellipsoid zone

jects were males and 22 were females, while in Ush2 group they were 18 and 28 , respectively. Table 1 summarizes the clinical data: the mean age of the whole cohort was 48.16 \pm 13.67 (52.32 \pm 12.94 in the arRP group and $44.08 \pm$ 13.24 in the Ush2 group). The disease onset was earlier in Usher patients as compared to those affected by nonsyndromic RP, irrespective of sex. Mean VA was $0.61 \pm 0.31$ and mean EZ width was $1,969.30 \pm 1,411.73 \mu \mathrm{m}$ in nonsyndromic patients, while mean VA was $0.49 \pm 0.30$ and mean EZ width was $1,306.63 \pm 1,246.49 \mu \mathrm{m}$ in syndromic ones.

This shows that VA and EZ width were significantly lower in Ush2 than in arRP patients $(p<0.0001$ and $p=$ 0.001 , respectively). This difference was statistically significant also when a model of analysis of covariance was applied to the dataset, using the criteria stated above.

ERM was detected in $34.8 \%(16 / 46)$ of arRP patients and in $65.2 \%(30 / 46)$ of Ush 2 patients $(p=0.003)$. A gender-related difference of ERM incidence was also detected: ERM was more frequent in males than in females, but

SD-OCT Analysis in USH2A-Associated

$\mathrm{RP}$ and Usher Syndrome it was not statistically significant. In the arRP group, the incidence of ERM was $37.5 \%(9 / 24)$ in males and $31.8 \%$ $(7 / 22)$ in females $(p=0.78)$, while in Ush2 group it was $77.8 \%(14 / 18)$ in males and $57.1 \%(16 / 28)$ in females $(p=$ $0.52)$.

CML was detected in $17.4 \%(8 / 46)$ of arRP patients and $30.4 \%(14 / 46)$ of Ush2 patients $(p=0.14)$. CML incidence in the arRP group was $20.8 \%(5 / 24)$ in males versus $13.6 \%(3 / 22)$ in females $(p=0.59)$, while in the Ush2 group it was $27.8 \%(5 / 18)$ in males versus $32.1 \%(9 / 28)$ in females $(p=0.82)$. We classified CML according to the grading system proposed by [16]: in the arRP group $12.5 \%$ $(1 / 8)$ of patients had mild CML, $50 \%(4 / 8)$ moderate, and $37.5 \%(3 / 8)$ severe. In the Ush2 group, $42.9 \%(6 / 14)$ of patients had mild CML, 50\% (7/14) moderate, and 7.1\% $(1 / 14)$ severe.

Overall, the genotype distribution by the expected variant impact on each allele was $27.2 \%$ high/high, $47.8 \%$ low/high, and 25\% low/low. The genotype distribution was statistically different $(p=0.0003)$ by dividing the 2 


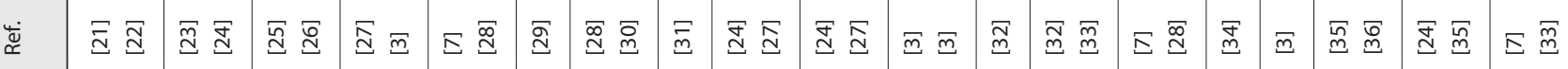

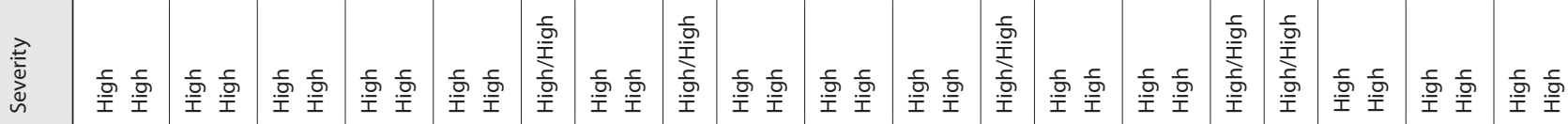

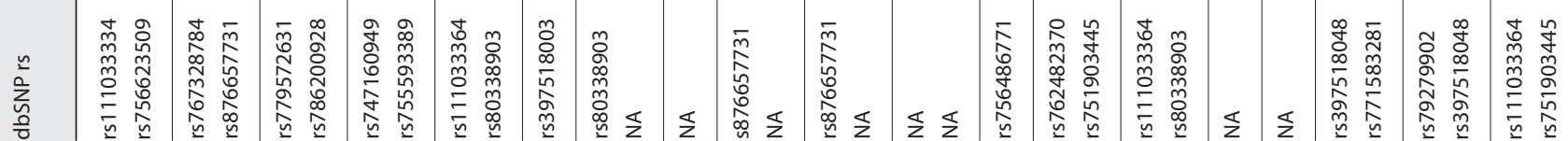

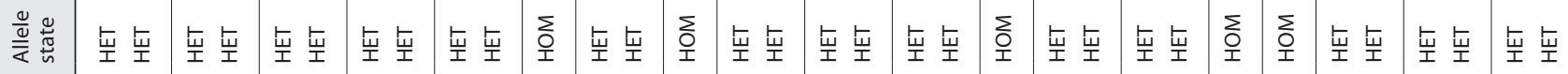

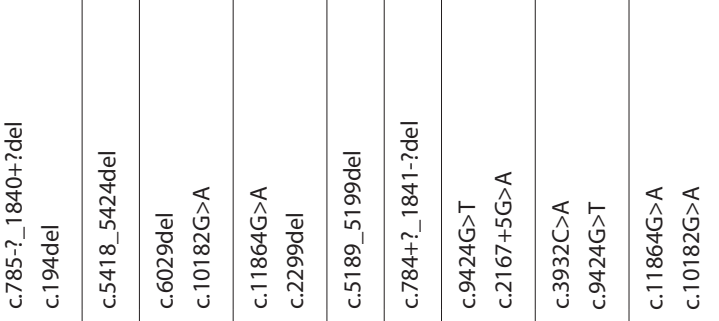




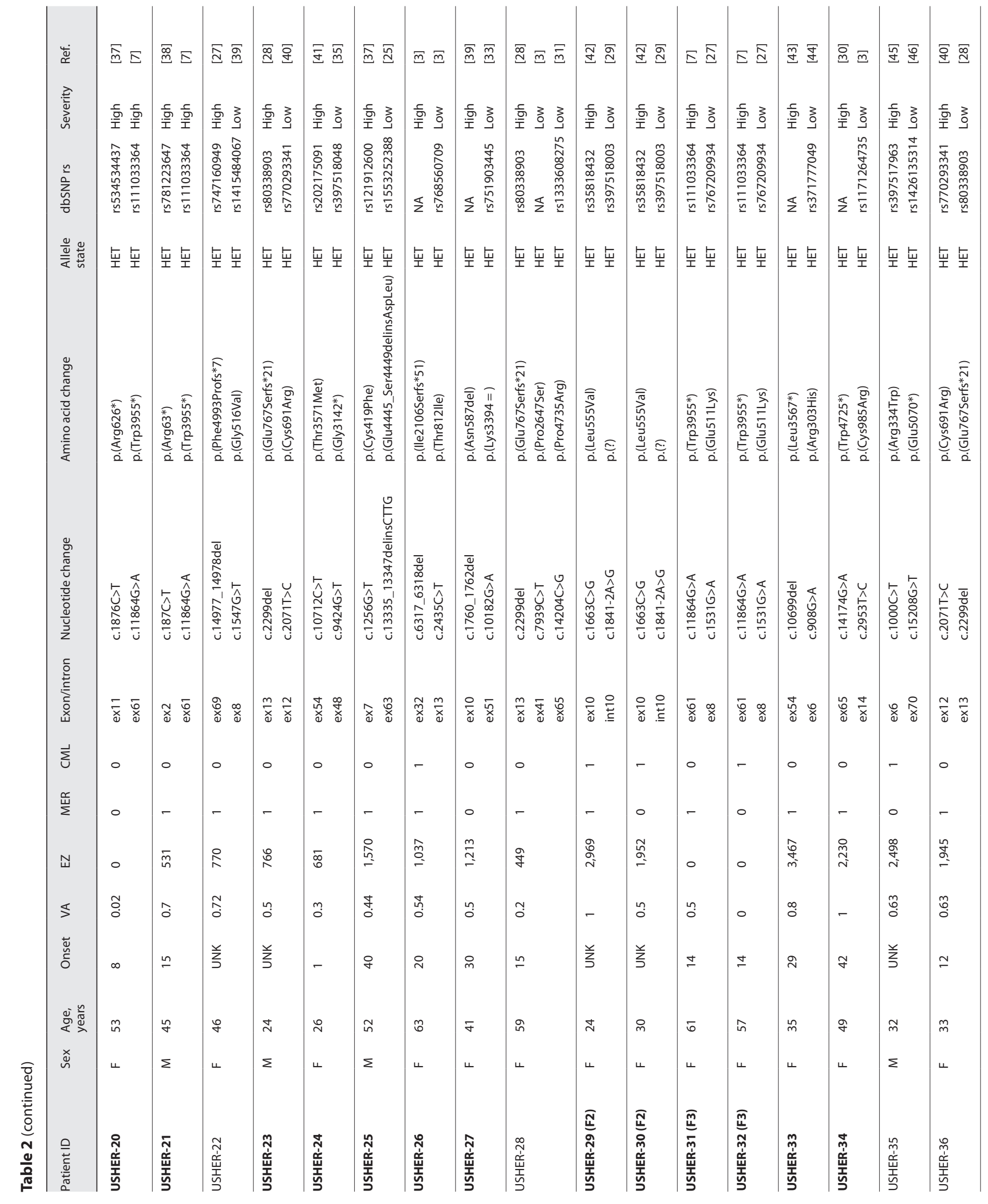




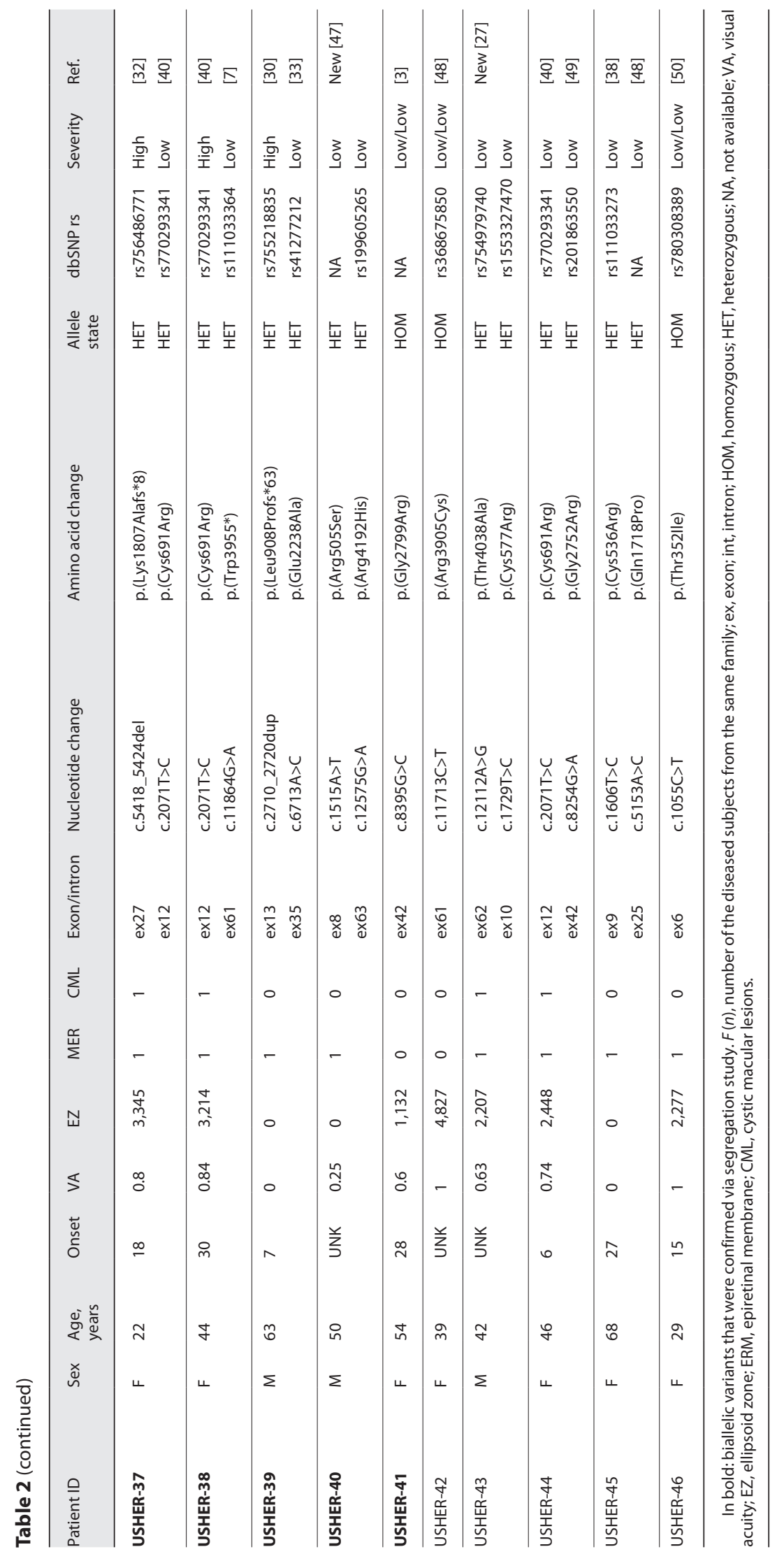




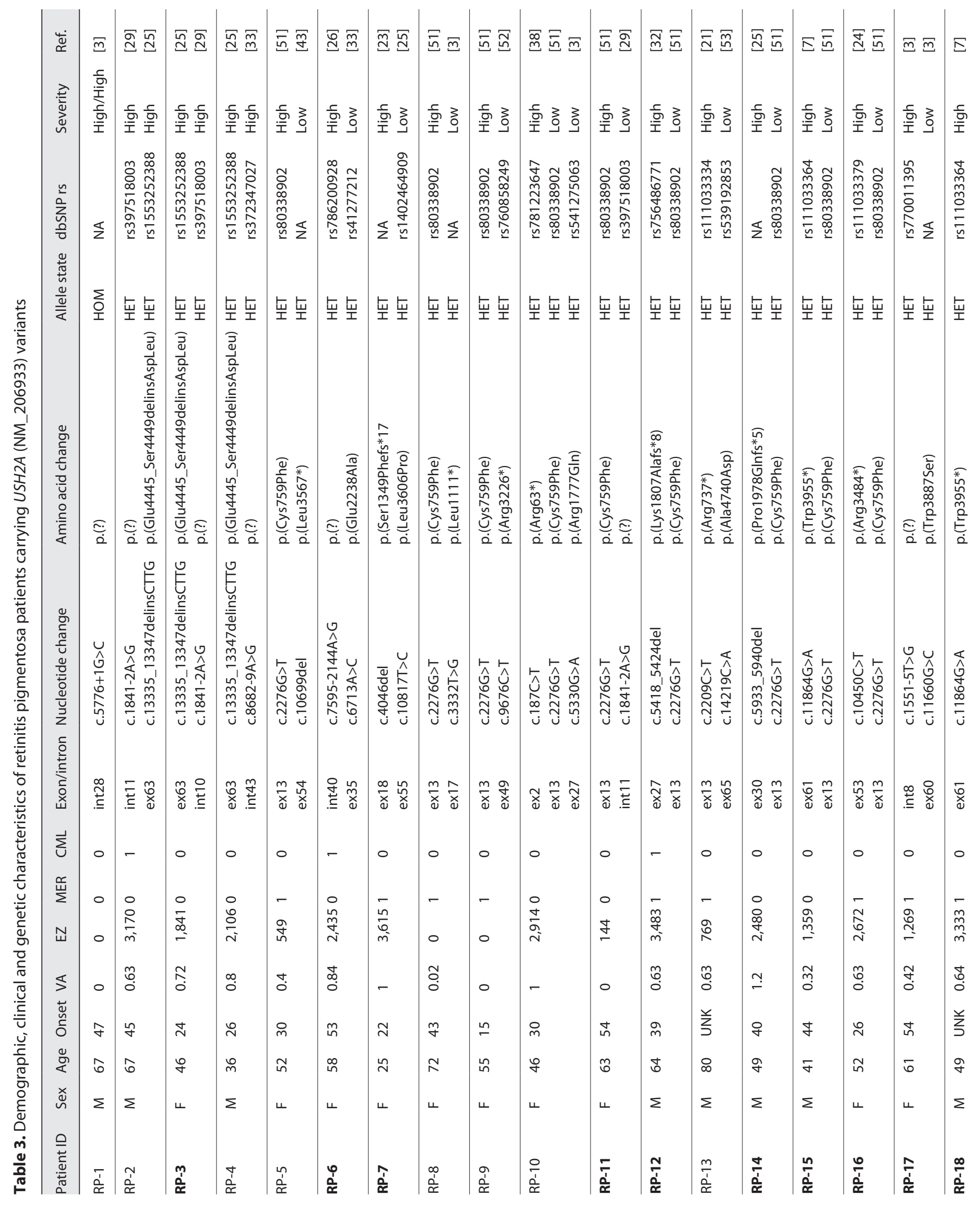




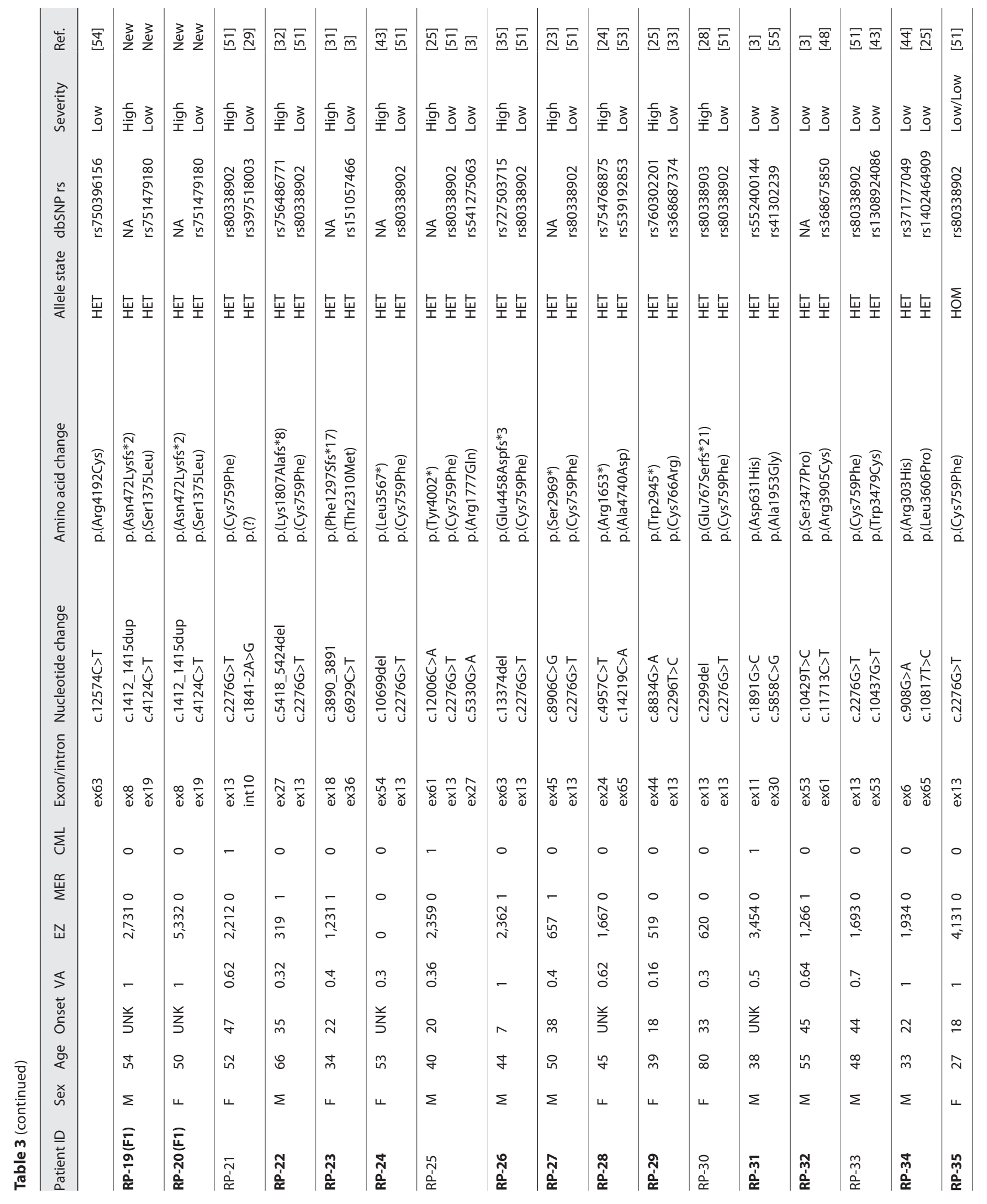




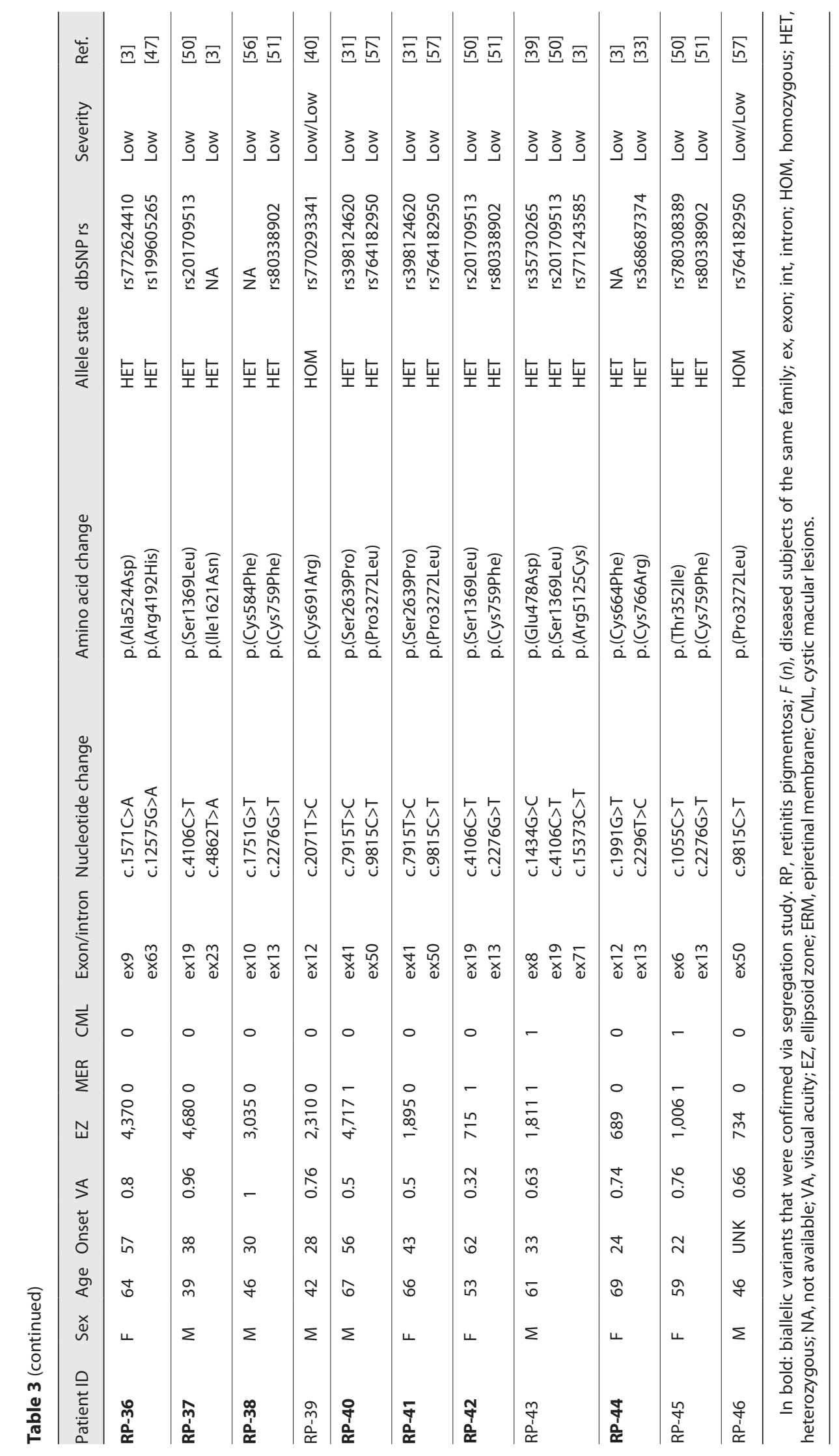


diseases: it was $45.7 \%$ high/high, 39.1\% low/high, and 15.2\% low/low for Usher patients (Table 2) and $8.7 \%$ high/high, 56.5\% low/high, and 34.8\% low/low for RP patients (Table 3). Considering the whole cohort of 92 subjects (46 arRP + 46 Ush2 patients), the genotype severity class significantly affected the parameters of VA and EZ width ( $p=0.004$ and $p=0.002$, respectively), while ERM and CML incidences were not affected $(p=0.26$ and $p=$ 0.86 , respectively).

The correlation between VA and EZ was substantial in the whole cohort $(r=0.76, p<0.0001)$ and in the high/ high $(r=0.68, p=0.01)$ and low/high groups $(r=0.74$, $p<0.0001)$; while it was moderate in the low/low group $(r=0.50, p=0.02)$ (shown in Fig. 2). About the onsets, the average ages of onset were distributed among the 3 groups (from earliest to latest) as follows: $22 \pm 10.8$ years in high/high patients, $28.5 \pm 14.4$ years in high/low patients, and $33.2 \pm 15.4$ years in low/low patients. The same statistics performed on the arRP and Ush2 groups, taken individually, did not reach significance in any of the considered parameters (data not shown).

\section{Discussion/Conclusion}

In our study, we evaluated macular structure using SD-OCT in a large cohort of USH2A-related arRP and Ush2 patients and found that syndromic cases show more advanced signs of retinal degeneration. As described in Materials and Methods section, we divided the patients into 3 categories (according to the severity of their variants) and found a significant correlation between their anatomical data and genotypes.

The differences in the disease course in syndromic versus nonsyndromic forms of USH2A-related retinal dystrophy were previously investigated: Sandberg et al. [58] compared VA, VF, and cone electroretinogram amplitudes in a large cohort of USH2A-related arRP and Ush2 and they did not find any statistically significant difference between the 2 groups for all the considered parameters. It is worth noting that the research compared only RP patients with the p.(Cys759Phe) - USH2A variant most frequently associated with the nonsyndromic disease - with Usher patients carrying the p.(Glu767Serfs*21) - the most common USH $2 A$ variant associated with the syndrome. About half of our RP patients $(21 / 46,45.6 \%)$ carried the p.(Cys759Phe) and $13 \%$ of our Usher syndrome patients carried the $\mathrm{p}$. (Glu767Serfs*21); however, in our population the most frequent Usher variant $(8 / 46,17.4 \%)$ was the p.(Trp3955*). Despite it could eliminate some confusion, the choice made in the study of Sandberg et al. [58] of comparing only 2 types of variants does not allow to generalize the result to other variants and/or populations.

Earlier impairment of retinal function in syndromic versus nonsyndromic USH2A patients was described by Pierrache et al. [30]; they found that Ush2 patients become visually impaired earlier, both according to VF and VA (13 and 18 years earlier, respectively). We analyzed the structural data in a different way: EZ width, as evaluated via SD-OCT, has become one of the most important markers to evaluate retinal involvement and to monitor the disease in IRDs services. EZ hyper-reflective band corresponds to the photoreceptor inner/outer segment junction: published literature shows the reproducibility and repeatability of EZ measurements and its correlation with functional data, like VA and VF [12-15, 59]. In our study, EZ width was significantly smaller in the Ush2 group than the arRP group, thus confirming that outer retina atrophy occurs at earlier age in the former rather than in the latter.

Consistently with EZ data, VA was found to be significantly lower in Ush2 than in arRP patients. A substantial correlation was also found between EZ width and VA $(p<0.0001)$, which has also been previously reported in RP patients by many authors [60-62]. Given the extreme variability of VF or ERG data, the wide spread of SD-OCT machines in ophthalmology clinics, and the straightforward acquisition and analysis of tomographic images, EZ width and integrity can be considered as the objective choice parameter to evaluate photoreceptors functionality.

Sengillo et al. [63] found in a small cohort of patients affected by USH2A-related retinal dystrophy that $30 \mathrm{~Hz}$ flicker responses were significantly higher in arRP patients than in Ush2 patients, thus confirming that Usher patients experience a faster decline of their visual functions. They also measured the EZ width in the 2 groups but, unlike in our study, the comparison did not reach statistical significance. We guess that the small number of patients and the younger age of their cohort could explain the differences with our findings.

We then assessed the presence of other macular abnormalities in our cohort of patients affected by USH $2 \mathrm{~A}$ related dystrophies: specifically, ERM and CML. In our dataset ERM was more prevalent than CML (50\% vs. $23.9 \%, p=0.0004)$; most importantly, we found an increased incidence of ERM in Ush2 patients compared to $\operatorname{arRP}(65.2 \%$ vs. $34.8 \%, p=0.0068)$. Many clinical studies investigated the prevalence of ERM in RP and Usher population, with results ranging from 0.6 to 
$80.5 \%$ [64-69]. This great variability could be due to at least 2 different factors. First of all, there is no universal consensus on the interpretation and classification of ERMs in SD-OCT scans: some authors include VMA and VMT as part of the ERMs group [70], but in other cases the presence of focal cells proliferation on the inner surface of the retina could be seen as the presence of ERMs by some OCT-readers, thus overestimating the incidence of such condition. Second, other factors such as age, inflammation, trauma, and previous ocular surgeries or laser treatments can play a role in ERM growth. Interestingly, in our cohort ERM was more prevalent in males rather than in females in both groups, but the difference was statistically significant only in the Ush2 group. Our results are in line with Testa et al. [66] in Ush2 but differ from another report by the same group in RP patients [67] and from literature about ERM incidence that indicates that for females the risk of developing ERM is higher [71].

On our daily practice experience, a small percentage of patients affected by arRP or Ush2 complicated by ERM requires vitreoretinal surgical procedures. Nevertheless, considering therapeutic surgical approaches such as gene therapy and artificial retina implant, ERM assessment plays a significant role in the preoperative evaluation of the patient.

As described above, we classified CML in both groups according to the grading system proposed by Sliesoraityte et al. [16]. Similarly to ERM, incidence of CML was higher in Ush2 rather than in arRP patients (30.4\% vs. $17.4 \%$, $p=0.2234)$; moreover, patients with arRP usually have a moderate or severe grade of CML (50 and $37.5 \%$, respectively, $p=0.3181$ ), while Ush2 patients usually have a mild or moderate grade of CML (42.9 and 50\%, respectively, $p=0.6357)$. Our data are in line with previous CML reports $[66,67,72-75]$, and have a strong correspondence with the one by Sliesoraityte et al. [16] in terms of incidence of CML in USH2A syndromic patients (30.4\% vs. $29 \%)$.

CML is a frequent issue for clinicians in IRDs services: there are no universally accepted guidelines available for the treatment of CML in both pediatric and adult population. Many strategies have been proposed - oral or topical administration of carbonic anhydrase inhibitors, antivegf agents, steroidal and anti-inflammatory treatments [76]. Recently published literature shows the efficacy of dexamethasone implants on CML reduction, but it is necessary to be cautious in considering this treatment: it is an invasive procedure that needs to be performed periodically and that can lead to possible complications, such as

SD-OCT Analysis in USH2A-Associated

$\mathrm{RP}$ and Usher Syndrome
IOP increase or cataract formation [77, 78]. To the best of our knowledge, this is the first comparison of the incidence of ERM and CML in syndromic and nonsyndromic USH $2 A$ patients. As described in detail in the Materials and Methods section, we divided the patients into 3 groups, according to severity of the variants we identified in USH $2 A$ gene.

Another research [30] attempted to associate USH2A variant types with visual prognosis in RP and Usher syndrome patients. In their report, patients were grouped based on the presence of truncating variants in their alleles, thus considering more severe the variants leading to a shortened or absent protein, the latter resulting from nonsense-mediated mRNA decay. Despite this little difference, the distribution of highly pathogenic variants was very similar to our findings for both Usher syndrome (high/high: $44 \%$ truncating/truncating vs. $45.7 \%$; low/ high: $40 \%$ nontruncating/truncating vs. $39.17 \%$; low/ low: $16 \%$ nontruncating/nontruncating vs. $15.2 \%)$ and RP patients (high/high: 3\% truncating/truncating vs. 8.7\%; low/high: $62 \%$ nontruncating/truncating vs. $56.5 \%$; and low/low: $36 \%$ nontruncating/nontruncating vs. $34.8 \%)$.

The results show that variant types and genotype classes are determining factors for the appearance of syndromic features. However, since the 3 genotypes can be found in both Usher and RP patients, other factors must necessarily play a determining role: among these could be the position of the variants at the protein level, which of the 2 usherin isoform is impacted by the variant, and the modifying influences of the genetic background in which the variant acts.

Considering the whole cohort, we found significant genotype-phenotype correlations: the 2 main clinical parameters evaluated, VA and EZ width, were substantially associated with the severity class of the variants. In particular, both EZ width and VA were lower in the high/ high group than in the low/high and low/low ones and the association was stronger in the low/high and high/high groups ( $p<0.0001$ and $p=0.01$, respectively) than in the low/low group $(p=0.02)$. While earlier VA decline in patients with truncating variants in USH2A gene has been previously reported [30], to the best of our knowledge, this is the first report of the association between EZ width and the severity of genetic variants in USH2A-related retinal dystrophies.

Additionally, we found that the disease onset occurs earlier in Usher patients, regardless of their genotype. Moreover, the genotype class was associated with both the age of onset - regardless of the disease - and the type 
of disease (i.e., Usher or RP). We can therefore conclude that the genotype influences both the age of onset of the disease and the possible manifestation of deafness, which distinguishes Usher syndrome from nonsyndromic RP.

New emerging therapies renewed enthusiasm in the field of IRDs and, on the other hand, pointed out the necessity to deeper understand disease mechanisms and to establish clinical parameters to assess the efficacy of therapies and to identify the patients that could benefit more from them. From this perspective, our study highlighted that retinal diseases, as evaluated by means of SD-OCT, show more advanced signs of degeneration in the syndromic rather than in the nonsyndromic form of retinal dystrophy related to USH2A gene, although the relationship between genetic variants and the resulting phenotype needs to be further investigated.

Our study has some limitations: the main concern is that we prospectively performed a single SD-OCT analysis, so we do not present follow-up data for each patient. This choice was made in order to have homogeneous data, since SD-OCT was not available for all patients and since in the past different OCT machines were used for clinical assessments. We know that we did not evaluate inter-individual disease progression; however, the 2 large cohorts considered allowed us to represent the differences in inter-group disease progression. Another drawback is that EZ width cannot be used as a parameter to monitor disease progression, both in the early stages - when EZ boundaries exceed the posterior pole (even if the advent of wide-field imaging is helping to overcome this limit) - and in the late stages - when outer retina is completely atrophic and EZ band is not easily detectable.

Overall, considering the number of homozygous patients and families studied by segregation analysis, the vast majority of our studied subjects $(73.9 \%)$ were confirmed to carry biallelic USH2A variants. However, we cannot exclude that at least some of the remaining patients included in this study carried in cis USH2A variants and they could not be considered genetically solved.

Furthermore, the severity classification of the variants, which considers only the missense as low-impact variants, is here extremely simplified. For example, lacking experimental evidence, we cannot be sure that noncanonical splice variants and in-frame variants could be considered to have the same high impact on protein function as truncating variants. However, these variants are very rare exceptions that do not affect the final result of this study.

\section{Acknowledgment}

The authors wish to thank Silvia Gaudenzi for her English language revision.

\section{Statement of Ethics}

The study was conducted according to the guidelines of the Declaration of Helsinki. This study protocol was reviewed and approved by the Ethical Committee of Azienda Sanitaria dell'Alto Adige, Italy, approval number 94-2016. Written informed consent has been obtained from the patients to publish this article.

\section{Conflict of Interest Statement}

The authors have no conflicts of interest to declare.

\section{Funding Sources}

This work was supported by funds from the Provincia Autonoma di Trento under initiative LP6/99 (dpg 1045/2017).

\section{Author Contributions}

L.C. and P.E.M. contributed to conceptualization; P.F., G.M., F.C., and M.P. contributed to methodology; G.M. and P.F. contributed to software; F.C. and M.P. contributed to validation; L.C., P.F., and P.E.M. contributed to formal analysis; L.C., D.R., A.M.M., and B.P. contributed to investigation; M.B. and L.R. contributed to resources; P.F., P.E.M., and B.P. contributed to data curation; L.C. and P.E.M. contributed to writing - original draft preparation; L.C., P.E.M., and M.C. contributed to writing - review and editing; M.C., M.B., and L.R. contributed to visualization; M.B., M.C., and L.R. contributed to supervision; L.C. contributed to project administration; M.B. contributed to funding acquisition. All authors have read and agreed to the published version of the manuscript.

\section{Data Availability Statement}

Clinical data reported in this work are available upon request from the corresponding authors. Results of patients' genetic analysis were reported in Colombo et al. [3], Invest Ophthalmol Vis Sci. 2021;62:13. 


\section{References}

1 Audo I, Bujakowska KM, Léveillard T, MohandSaïd S, Lancelot ME, Germain A, et al. Development and application of a next-generation-sequencing (NGS) approach to detect known and novel gene defects underlying retinal diseases. Orphanet J Rare Dis. 2012 Jan 25;7:8.

2 Neveling K, Collin RW, Gilissen C, van Huet RA, Visser L, Kwint MP, et al. Next-generation genetic testing for retinitis pigmentosa. Hum Mutat. 2012 Jun;33(6):963-72.

3 Colombo L, Maltese PE, Castori M, El Shamieh S, Zeitz C, Audo I, et al. Molecular epidemiology in 591 Italian probands with nonsyndromic retinitis pigmentosa and usher syndrome. Invest Ophthalmol Vis Sci. 2021 Feb 1;62(2):13.

4 Martin-Merida I, Avila-Fernandez A, Del Pozo-Valero M, Blanco-Kelly F, Zurita O, PerezCarro R, et al. Genomic landscape of sporadic retinitis pigmentosa: findings from $877 \mathrm{Span}-$ ish cases. Ophthalmology. 2019 Aug;126(8): $1181-8$.

5 Reiners J, Nagel-Wolfrum K, Jürgens K, Märker T, Wolfrum U. Molecular basis of human Usher syndrome: deciphering the meshes of the Usher protein network provides insights into the pathomechanisms of the Usher disease. Exp Eye Res. 2006 Jul;83(1):97-119.

6 Bhattacharya G, Miller C, Kimberling WJ, Jablonski MM, Cosgrove D. Localization and expression of usherin: a novel basement membrane protein defective in people with Usher's syndrome type IIa. Hear Res. 2002 Jan;163(1-2):1-11.

7 van Wijk E, Pennings RJ, te Brinke $\mathrm{H}$, Claassen A, Yntema HG, Hoefsloot LH, et al. Identification of 51 novel exons of the Usher syndrome type $2 \mathrm{~A}$ (USH2A) gene that encode multiple conserved functional domains and that are mutated in patients with Usher syndrome type II. Am J Hum Genet. 2004 Apr; 74(4):738-44

8 Liu X, Bulgakov OV, Darrow KN, Pawlyk B, Adamian M, Liberman MC, et al. Usherin is required for maintenance of retinal photoreceptors and normal development of cochlear hair cells. Proc Natl Acad Sci U S A. 2007 Mar 13;104(11):4413-8.

9 Mills JO, Jalil A, Stanga PE. Electronic retinal implants and artificial vision: journey and present. Eye. 2017 Oct;31(10):1383-98.

10 Tang Z, Zhang Y, Wang Y, Zhang D, Shen B, Luo M, et al. Progress of stem/progenitor cellbased therapy for retinal degeneration. J Transl Med. 2017 May 10;15(1):99.

11 Ziccardi L, Cordeddu V, Gaddini L, Matteucci A, Parravano M, Malchiodi-Albedi F, et al. Gene therapy in retinal dystrophies. Int J Mol Sci. 2019 Nov 14;20(22):5722.

12 Birch DG, Locke KG, Wen Y, Locke KI, Hoffman DR, Hood DC. Spectral-domain optical coherence tomography measures of outer segment layer progression in patients with $\mathrm{X}$ linked retinitis pigmentosa. JAMA Ophthalmol. 2013 Sep;131(9):1143-50.
13 Cai CX, Locke KG, Ramachandran R, Birch DG, Hood DC. A comparison of progressive loss of the ellipsoid zone (EZ) band in autosomal dominant and $\mathrm{x}$-linked retinitis pigmentosa. Invest Ophthalmol Vis Sci. 2014 Oct 23; 55(11):7417-22.

14 Sujirakul T, Lin MK, Duong J, Wei Y, LopezPintado S, Tsang SH. Multimodal imaging of central retinal disease progression in a 2-year mean follow-up of retinitis pigmentosa. Am J Ophthalmol. 2015 Oct;160(4):786-98.e4.

15 Hariri AH, Zhang HY, Ho A, Francis P, Weleber RG, Birch DG, et al. Quantification of ellipsoid zone changes in retinitis pigmentosa using en face spectral domain-optical coherence tomography. JAMA Ophthalmol. 2016 Jun 1;134(6):628-35.

16 Sliesoraityte I, Peto T, Mohand-Said S, Sahel JA. Novel grading system for quantification of cystic macular lesions in Usher syndrome. Orphanet J Rare Dis. 2015 Dec 10;10:157.

17 Maltese PE, Orlova N, Krasikova E, Emelyanchik E, Cheremisina A, Kuscaeva A, et al. Gene-targeted analysis of clinically diagnosed long QT Russian families. Int Heart J. 2017 Feb 7;58(1):81-7.

18 Marceddu G, Dallavilla T, Guerri G, Manara E, Chiurazzi P, Bertelli M. PipeMAGI: an integrated and validated workflow for analysis of NGS data for clinical diagnostics. Eur Rev Med Pharmacol Sci. 2019 Aug;23(15):675365.

19 Richards S, Aziz N, Bale S, Bick D, Das S, Gastier-Foster J, et al. Standards and guidelines for the interpretation of sequence variants: a joint consensus recommendation of the American College of Medical Genetics and Genomics and the Association for Molecular Pathology. Genet Med. 2015 May; 17(5):405-24.

20 Kopanos C, Tsiolkas V, Kouris A, Chapple CE, Albarca Aguilera M, Meyer R, et al. VarSome: the human genomic variant search engine. Bioinformatics. 2019 Jun 1;35(11): 1978-80.

21 Kaiserman N, Obolensky A, Banin E, Sharon D. Novel USH2A mutations in Israeli patients with retinitis pigmentosa and Usher syndrome type 2. Arch Ophthalmol. 2007 Feb; 125(2):219-24.

22 Maubaret C, Griffoin JM, Arnaud B, Hamel C. Novel mutations in MYO7A and USH2A in Usher syndrome. Ophthalmic Genet. 2005 Mar;26(1):25-9.

23 Sodi A, Mariottini A, Passerini I, Murro V, Tachyla I, Bianchi B, et al. MYO7A and USH2A gene sequence variants in Italian patients with Usher syndrome. Mol Vis. 2014 Dec 23;20:1717-31.

24 Dreyer B, Brox V, Tranebjaerg L, Rosenberg T, Sadeghi AM, Möller C, et al. Spectrum of USH2A mutations in Scandinavian patients with Usher syndrome type II. Hum Mutat. 2008 Mar;29(3):451.
25 McGee TL, Seyedahmadi BJ, Sweeney MO, Dryja TP, Berson EL. Novel mutations in the long isoform of the USH2A gene in patients with Usher syndrome type II or non-syndromic retinitis pigmentosa. J Med Genet. 2010 Jul;47(7):499-506.

26 Vaché C, Besnard T, le Berre P, García-García G, Baux D, Larrieu L, et al. Usher syndrome type 2 caused by activation of an USH2A pseudoexon: implications for diagnosis and therapy. Hum Mutat. 2012 Jan;33(1):104-8.

27 Baux D, Blanchet C, Hamel C, Meunier I, Larrieu L, Faugère V, et al. Enrichment of LOVDUSHbases with 152 USH2A genotypes defines an extensive mutational spectrum and highlights missense hotspots. Hum Mutat. 2014 Oct;35(10):1179-86.

28 Eudy JD, Weston MD, Yao S, Hoover DM, Rehm HL, Ma-Edmonds M, et al. Mutation of a gene encoding a protein with extracellular matrix motifs in Usher syndrome type IIa. Science. 1998 Jun 12;280(5370):1753-7.

29 Bernal S, Ayuso C, Antiñolo G, Gimenez A, Borrego S, Trujillo MJ, et al. Mutations in USH2A in Spanish patients with autosomal recessive retinitis pigmentosa: high prevalence and phenotypic variation. J Med Genet. 2003 Jan;40(1):e8.

30 Pierrache LH, Hartel BP, van Wijk E, MeesterSmoor MA, Cremers FP, de Baere E, et al. Visual prognosis in USH2A-associated retinitis pigmentosa is worse for patients with Usher syndrome type IIa than for those with nonsyndromic retinitis pigmentosa. Ophthalmology. 2016 May;123(5):1151-60.

31 Bonnet C, Riahi Z, Chantot-Bastaraud S, Smagghe L, Letexier M, Marcaillou C, et al. An innovative strategy for the molecular diagnosis of Usher syndrome identifies causal biallelic mutations in $93 \%$ of European patients. Eur J Hum Genet. 2016 Dec;24(12): $1730-8$.

32 Lenarduzzi S, Vozzi D, Morgan A, Rubinato E, D'Eustacchio A, Osland TM, et al. Usher syndrome: an effective sequencing approach to establish a genetic and clinical diagnosis. Hear Res. 2015 Feb;320:18-23.

33 Glöckle N, Kohl S, Mohr J, Scheurenbrand T, Sprecher A, Weisschuh N, et al. Panel-based next generation sequencing as a reliable and efficient technique to detect mutations in unselected patients with retinal dystrophies. Eur J Hum Genet. 2014 Jan;22(1):99-104.

34 Bonnet C, Grati M, Marlin S, Levilliers J, Hardelin JP, Parodi M, et al. Complete exon sequencing of all known Usher syndrome genes greatly improves molecular diagnosis. Orphanet J Rare Dis. 2011 May 11;6:21.

35 Baux D, Larrieu L, Blanchet C, Hamel C, Ben Salah S, Vielle A, et al. Molecular and in silico analyses of the full-length isoform of usherin identify new pathogenic alleles in Usher type II patients. Hum Mutat. 2007 Aug;28(8):7819. 
36 Nájera C, Beneyto M, Blanca J, Aller E, Fontcuberta A, Millán JM, et al. Mutations in myosin VIIA (MYO7A) and usherin (USH2A) in Spanish patients with Usher syndrome types I and II, respectively. Hum Mutat. 2002 Jul; 20(1):76-7.

37 Weston MD, Eudy JD, Fujita S, Yao S, Usami $\mathrm{S}$, Cremers C, et al. Genomic structure and identification of novel mutations in usherin, the gene responsible for Usher syndrome type IIa. Am J Hum Genet. 2000 Apr;66(4):1199_ 210.

38 Dreyer B, Tranebjaerg L, Rosenberg T, Weston MD, Kimberling WJ, Nilssen $\mathrm{O}$. Identification of novel USH2A mutations: implications for the structure of USH2A protein. Eur J Hum Genet. 2000 Jul;8(7):500-6.

39 Seyedahmadi BJ, Rivolta C, Keene JA, Berson EL, Dryja TP. Comprehensive screening of the USH2A gene in Usher syndrome type II and non-syndromic recessive retinitis pigmentosa. Exp Eye Res. 2004 Aug;79(2):16773.

40 Pierrottet CO, Zuntini M, Digiuni M, Bazzanella I, Ferri P, Paderni R, et al. Syndromic and non-syndromic forms of retinitis pigmentosa: a comprehensive Italian clinical and molecular study reveals new mutations. Genet Mol Res. 2014 Oct 27;13(4):8815-33.

41 Aller E, Jaijo T, Beneyto M, Nájera C, Oltra S, Ayuso C, et al. Identification of 14 novel mutations in the long isoform of USH2A in Spanish patients with Usher syndrome type II. J Med Genet. 2006 Nov;43(11):e55.

42 Leroy BP, Aragon-Martin JA, Weston MD, Bessant DA, Willis C, Webster AR, et al. Spectrum of mutations in USH2A in British patients with Usher syndrome type II. Exp Eye Res. 2001 May;72(5):503-9.

43 Galli-Resta L, Placidi G, Campagna F, Ziccardi L, Piccardi M, Minnella A, et al. Central retina functional damage in Usher syndrome type 2: 22 years of focal macular ERG analysis in a patient population from Central and Southern Italy. Invest Ophthalmol Vis Sci. 2018 Aug 1;59(10):3827-35.

44 Yan D, Ouyang X, Patterson DM, Du LL, Jacobson SG, Liu XZ. Mutation analysis in the long isoform of USH2A in American patients with Usher Syndrome type II. J Hum Genet. 2009 Dec;54(12):732-8.

45 Adato A, Weston MD, Berry A, Kimberling WJ, Bonne-Tamir A. Three novel mutations and twelve polymorphisms identified in the USH2A gene in Israeli USH2 families. Hum Mutat. 2000 Apr;15(4):388.

46 Eandi CM, Dallorto L, Spinetta R, Micieli MP, Vanzetti M, Mariottini A, et al. Targeted next generation sequencing in Italian patients with Usher syndrome: phenotype-genotype correlations. Sci Rep. 2017 Nov 15;7(1):15681.

47 Ávila-Fernández A, Cantalapiedra D, Aller E, Vallespín E, Aguirre-Lambán J, Blanco-Kelly F, et al. Mutation analysis of 272 Spanish families affected by autosomal recessive retinitis pigmentosa using a genotyping microarray. Mol Vis. 2010 Dec 3;16:2550-8.
48 Krawitz PM, Schiska D, Krüger U, Appelt S, Heinrich V, Parkhomchuk D, et al. Screening for single nucleotide variants, small indels and exon deletions with a next-generation sequencing based gene panel approach for Usher syndrome. Mol Genet Genomic Med. 2014 Sep;2(5):393-401.

49 Nakanishi H, Ohtsubo M, Iwasaki S, Hotta Y, Mizuta K, Mineta $\mathrm{H}$, et al. Identification of 11 novel mutations in USH2A among Japanese patients with Usher syndrome type 2. Clin Genet. 2009 Oct;76(4):383-91.

50 Cremers FP, Kimberling WJ, Külm M, de Brouwer AP, van Wijk E, te Brinke $\mathrm{H}$, et al. Development of a genotyping microarray for Usher syndrome. J Med Genet. 2007 Feb; 44(2):153-60.

51 Rivolta C, Sweklo EA, Berson EL, Dryja TP. Missense mutation in the USH2A gene: association with recessive retinitis pigmentosa without hearing loss. Am J Hum Genet. 2000 Jun;66(6): 1975-8.

52 Katagiri S, Akahori M, Sergeev Y, Yoshitake $\mathrm{K}$, Ikeo K, Furuno M, et al. Whole exome analysis identifies frequent CNGA1 mutations in Japanese population with autosomal recessive retinitis pigmentosa. PLoS One. 2014 Sep 30;9(9):e108721.

53 Licastro D, Mutarelli M, Peluso I, Neveling K, Wieskamp N, Rispoli R, et al. Molecular diagnosis of Usher syndrome: application of two different next generation sequencing-based procedures. PLoS One. 2012;7(8):e43799.

54 Corton M, Nishiguchi KM, Avila-Fernández A, Nikopoulos K, Riveiro-Alvarez R, Tatu SD, et al. Exome sequencing of index patients with retinal dystrophies as a tool for molecular diagnosis. PLoS One. 2013 Jun 14;8(6):e65574.

55 Chebil A, Falfoul Y, Habibi I, Munier F, Schorderet D, El Matri L. Corrélations phénotype-génotype de la rétinopathie pigmentaire non syndromique : à propos de dix familles tunisiennes [Genotype-phenotype correlation in ten Tunisian families with non-syndromic retinitis pigmentosa]. J Fr Ophtalmol. 2016 Mar;39(3):277-86.

56 de Castro-Miró M, Pomares E, Lorés-Motta L, Tonda R, Dopazo J, Marfany G, et al. Combined genetic and high-throughput strategies for molecular diagnosis of inherited retinal dystrophies. PLoS One. 2014 Feb 7;9(2): e88410.

57 Herrera W, Aleman TS, Cideciyan AV, Roman AJ, Banin E, Ben-Yosef T, et al. Retinal disease in Usher syndrome III caused by mutations in the clarin-1 gene. Invest Ophthalmol Vis Sci. 2008 Jun;49(6):2651-60.

58 Sandberg MA, Rosner B, Weigel-DiFranco C, McGee TL, Dryja TP, Berson EL. Disease course in patients with autosomal recessive retinitis pigmentosa due to the USH2A gene. Invest Ophthalmol Vis Sci. 2008 Dec;49(12): 5532-9.
59 Lima LH, Burke T, Greenstein VC, Chou CL, Cella W, Yannuzzi LA, et al. Progressive constriction of the hyperautofluorescent ring in retinitis pigmentosa. Am J Ophthalmol. 2012 Apr;153(4):718-27, 727.e1-2.

60 Aizawa S, Mitamura Y, Hagiwara A, Sugawara T, Yamamoto S. Changes of fundus autofluorescence, photoreceptor inner and outer segment junction line, and visual function in patients with retinitis pigmentosa. Clin Exp Ophthalmol. 2010 Aug;38(6):597-604.

61 Mitamura Y, Mitamura-Aizawa S, Katome T, Naito T, Hagiwara A, Kumagai K, et al. Photoreceptor impairment and restoration on optical coherence tomographic image. J Ophthalmol. 2013;2013:518170.

62 Liu G, Li H, Liu X, Xu D, Wang F. Structural analysis of retinal photoreceptor ellipsoid zone and postreceptor retinal layer associated with visual acuity in patients with retinitis pigmentosa by ganglion cell analysis combined with OCT imaging. Medicine. 2016 Dec;95(52):e5785.

63 Sengillo JD, Cabral T, Schuerch K, Duong J, Lee W, Boudreault K, et al. Electroretinography reveals difference in cone function between syndromic and nonsyndromic USH2A patients. Sci Rep. 2017 Sep 11;7(1):11170.

64 Fishman GA, Fishman M, Maggiano J. Macular lesions associated with retinitis pigmentosa. Arch Ophthalmol. 1977 May;95(5):798803.

65 Hagiwara A, Yamamoto S, Ogata K, Sugawara T, Hiramatsu A, Shibata M, et al. Macular abnormalities in patients with retinitis pigmentosa: prevalence on OCT examination and outcomes of vitreoretinal surgery. Acta Ophthalmol. 2011 Mar;89(2):e122-5.

66 Testa F, Melillo P, Rossi S, Marcelli V, de Benedictis A, Colucci R, et al. Prevalence of macular abnormalities assessed by optical coherence tomography in patients with Usher syndrome. Ophthalmic Genet. 2018 Jan-Feb; 39(1):17-21.

67 Testa F, Rossi S, Colucci R, Gallo B, Di Iorio $\mathrm{V}$, della Corte M, et al. Macular abnormalities in Italian patients with retinitis pigmentosa. Br J Ophthalmol. 2014 Jul;98(7):946-50.

68 Colombo L, Montesano G, Sala B, Patelli F, Maltese P, Abeshi A, et al. Comparison of 5 -year progression of retinitis pigmentosa involving the posterior pole among siblings by means of SD-OCT: a retrospective study. BMC Ophthalmol. 2018 Jun 26;18(1):153.

69 Fragiotta S, Rossi T, Carnevale C, Cutini A, Tricarico S, Casillo L, et al. Vitreo-macular interface disorders in retinitis pigmentosa. Graefes Arch Clin Exp Ophthalmol. 2019 Oct; 257(10):2137-46

70 Konidaris V, Androudi S, Alexandridis A, Dastiridou A, Brazitikos P. Optical coherence tomography-guided classification of epiretinal membranes. Int Ophthalmol. 2015 Aug; 35(4):495-501. 
71 Xiao W, Chen X, Yan W, Zhu Z, He M. Prevalence and risk factors of epiretinal membranes: a systematic review and meta-analysis of population-based studies. BMJ Open. 2017 Sep 25;7(9):e014644.

72 Adackapara CA, Sunness JS, Dibernardo CW, Melia BM, Dagnelie G. Prevalence of cystoid macular edema and stability in oct retinal thickness in eyes with retinitis pigmentosa during a 48-week lutein trial. Retina. 2008 Jan; 28(1):103-10.

73 Hirakawa H, Iijima H, Gohdo T, Tsukahara S. Optical coherence tomography of cystoid macular edema associated with retinitis pigmentosa. Am J Ophthalmol. 1999 Aug;128(2): $185-91$.
74 Chung H, Hwang JU, Kim JG, Yoon YH. Optical coherence tomography in the diagnosis and monitoring of cystoid macular edema in patients with retinitis pigmentosa. Retina. 2006 Oct;26(8):922-7.

75 Makiyama Y, Oishi A, Otani A, Ogino K, Nakagawa S, Kurimoto M, et al. Prevalence and spatial distribution of cystoid spaces in retinitis pigmentosa: investigation with spectral domain optical coherence tomography. Retina. 2014 May;34(5):981-8.

76 Bakthavatchalam M, Lai FHP, Song Rong S, $\mathrm{Ng}$ DS, Brelen ME. Treatment of cystoid macular edema secondary to retinitis pigmentosa: a systematic review. Surv Ophthalmol. 2018 May-Jun;63(3):329-39.
77 Mansour AM, Sheheitli H, Kucukerdonmez C, Sisk RA, Moura R, Moschos MM, et al. Intravitreal dexamethasone implant in retinitis pigmentosa-related cystoid macular edema. Retina. 2018 Feb;38(2):416-23.

78 Veritti D, Sarao V, De Nadai K, Chizzolini M, Parmeggiani F, Perissin L, et al. Dexamethasone implant produces better outcomes than oral acetazolamide in patients with cystoid macular edema secondary to retinitis pigmentosa. J Ocul Pharmacol Ther. 2020 Apr; 36(3):190-7. 